\title{
Erratum
}

\section{Inheritance of Electrophoretic Variants of Tuber Proteins in Solanum tuberosum Haploids ${ }^{1}$}

P. W. Simon and S. J. Peloquin

Left and right patterns should be reversed in Figure 1 to correspond to the legend, and W231 information (p. 1059) should accompany Figure 2.

\footnotetext{
'Biochemical Genetics, (1980) 18:1055.
} 